\author{
Christophe Dornier \\ G. Aernout Somsen \\ Marko K. Ivancevic \\ Nael F. Osman \\ Dominique Didier \\ Alberto Righetti \\ Jean-Paul Vallée
}

\section{Comparison between tagged MRI and standard cine MRI for evaluation of left ventricular ejection fraction}

Received: 18 July 2003

Revised: 17 February 2004

Accepted: 8 March 2004

Published online: 6 April 2004

(C) Springer-Verlag 2004

\begin{abstract}
Global left ventricular function is a prognostic indicator and is used to evaluate therapeutical interventions in patients with heart failure. Regional left ventricular function can be determined with tagged MRI. Assessment of global left ventricular function using the tagging data may have additional clinical value without incurring extra scanning time, which is currently a limiting factor in cardiac imaging. Direct determination of end-diastolic volume is not possible with conventional tagged MRI. However, end-systolic volume can be directly measured because myocardium-blood contrast improves through a tagged image series. We investigated the potential of tagged MRI using frequency-domain analysis software to retrospectively track end-diastolic contour from endsystolic contour and subsequently calculate the ejection fraction. Tagged MRI was compared with the standard bright-blood cine MRI in healthy volunteers $(n=20)$ and patients with previous myocardial infarction $(n=8)$. Left ventricular ejection fraction de-
\end{abstract}

rived from tagged MRI is linearly correlated to left ventricular ejection fraction obtained by standard cardiac cine MRI $(y=1.0 x+1.31, r>0.98$, $p=0.014$ ). In addition, the inter-observer and intra-observer coefficient of variation for left ventricular ejection fraction measurements was low $\left(\mathrm{CV}_{\text {intra }}=0.4 \%, \mathrm{CV}_{\text {inter }}=1.3 \%\right)$. With tagged MRI, only end-systolic volume needs to be manually determined, and accurate estimation of left ventricular ejection fraction is obtained because end-diastolic and end-systolic volumes are determined using identical anatomical points. Our data indicate that tagged MRI can be used to quantitatively assess both regional and global left ventricular function. Therefore, tagged MRI may be a valuable clinical tool for determining the prognosis and evaluating the effect of therapeutical intervention using a single imaging session in patients with left ventricular dysfunction.

Keywords Cardiac imaging . Tagged MRI - Ejection fraction · Cine MRI $\cdot$ Left ventricle

\section{Introduction}

The prevalence and incidence of left ventricular (LV) dysfunction is increasing in the Western world, which can be attributed to improved treatment and subsequent increased survival of both acute myocardial infarct and heart failure patients. Since global LV function, expressed as left ventricular ejection fraction (LV-EF), is an important prognostic indicator [1,2] and can be used to evaluate the effect of therapeutic interventions and since regional LV function can be used to identify viable myocardium [3, 4], a reliable technique for determining both global and regional LV function is needed.

Cine MRI is highly accurate for determining global myocardial function without using ionising radiation or assumptions of ventricular shape, which are major draw- 
backs in alternative imaging modalities such as nuclear ventriculography [5] and echocardiography [6], respectively. However, quantitative analysis of regional myocardial function cannot be performed.

Tagged MRI is an accepted method for assessment of regional myocardial function $[7,8]$. This technique is based on the cine MRI sequence and induces fiducial markers in the myocardium that can be followed during cardiac contraction. The technique consists of the application of a saturation pattern (i.e. dark lines) placed in the myocardial tissue using spatially selective radiofrequency pulses prior to application of the imaging sequence $[9,10]$. Since there is poor contrast between the LV cavity and the myocardium just after tag preparation, measurement of LV volumes has been considered unreliable. However, later in the image series, motion of the blood during the cardiac cycle improves the blood-myocardium contrast, which may enable adequate volume assessment by tracking the endocardial contour placed on the end-systolic frame over all the cardiac frames.

The aim of this study was to evaluate tagged MRI for the assessment of global myocardial function, which can be used as a prognostic indicator and for evaluating therapeutic intervention. The acquisition of both global and regional $\mathrm{LV}$ function in one imaging session will ensure optimal use of available scanning time, which is often regarded as a practical limitation in clinical cardiac MRI imaging, especially in severely ill patients.

\section{Materials and methods}

\section{Subjects}

Twenty healthy volunteers and eight patients with previous myocardial infarction were examined with both tagged MRI and cine MRI. None of the healthy subjects had a history of cardiovascular disease or any risk factor for cardiovascular disease. In all studied individuals, there were no contraindications for an MRI examinations. The Medical Ethical Committee of our institution approved this study and all individuals gave informed consent.

\section{Acquisition protocol}

Imaging was performed on an Eclipse 1.5-T MR system (Philips Medical Systems, Cleveland, $\mathrm{OH}$ ) equipped with a cardiac phased-array coil. Both tagged and cine sequences were acquired during breath-hold. The protocol consisted of multiple short-axis views (6-8 adjacent slices) acquired with the tagged and the standard cine sequence. The total acquisition time was about $35 \mathrm{~min}$.

\section{Tagged MRI}

The tagged sequence consisted of a 1-3-3-1 binomial pulse tagging preparation block, followed by a FAST gradient-echo imaging block [11].

The imaging parameters were: tag flip angle, $90^{\circ}$; tag spacing, $12 \mathrm{~mm}$; tag modulation was along read direction; pulse repetition time (TR)/echo time (TE), $7.6 / 2.6 \mathrm{~ms}$; flip angle, $15^{\circ}$; sampling bandwidth, $31.25 \mathrm{kHz}$; phase encoding group (PEG) size, 6-8 according to the patient's heart rate; slice thickness, $8 \mathrm{~mm}$; field of view (FOV) adapted to the patient's size, $64 \times 256$ acquisition matrix, zero filled by a factor of two during reconstruction; electrocardiogram (ECG) gating with 12 phases covering $65 \%$ of the cardiac cycle. To perform a two-dimensional (2D) heart analysis, two sequences with perpendicular tag directions were acquired during the same breath-hold [12].

\section{Cine MRI}

The parameters for the standard FAST gradient-echo cine sequence were: TR/TE, 9.5/3.3 ms; flip angle, $35^{\circ}$; sampling bandwidth, $25 \mathrm{kHz}$; PEG size, 6-8 according to heart rate; slice thickness, $8 \mathrm{~mm}, 128 \times 256$ matrix, zero filled by a factor of two during reconstruction; ECG gating with 14 phases covering $88 \%$ of the cardiac cycle.

Image analysis

The standard multislice short-axis cine MRI series were analysed using the ImageJ program (National Institutes of Health, Bethesda, MD, http://rsb.info.nih.gov/ij/). The endocardial surfaces of the LV were traced manually. The window and level settings of a representative midventricular image were optimised for best image contrast between the myocardium and the ventricle and then set for all images. End-diastolic (ED) images were chosen as the first phase after triggering off the R-wave. End-systolic (ES) images were defined as the images with the smallest cavity area.

The tagged MRI data were analysed with HARP software [13] based on harmonic phase imaging for automated quantification of myocardial strains. The endocardial border just inside the myocardium (1-2 mm) was manually traced (as a closed polygon) in the ES frame and then tracked over the cardiac cycle (see Fig. 1). ED images and ES images were defined in the same way as above.

The volume of each slice was determined using the area within the endocardial tracing multiplied by the slice thickness. The papillary muscles were regarded as being part of the LV cavity [14, 15]. The end-diastolic volume (EDV) and end-systolic volume (ESV) were calculated by summing the volumes of all short-axis slices. The EF was calculated as (EDV - ESV)/EDV.

\section{Statistical analysis}

Mean values and standard deviations were calculated for all parameters. Agreement between tagged MRI and standard cine MRI data was evaluated according to the method described by Bland and Altman [16]. In addition, linear regression analysis was used to compare corresponding measurements from the different techniques and a paired student's $t$ test was performed to determine significant differences between results with a probability level of $p<0.05$ considered as significant.

Intra-observer and inter-observer agreements were assessed by processing all the tagged images twice by the same observer (interval of 4 months) and by two different observers using correlation and coefficient of variation $(\mathrm{CV})$.

\section{Results}

This study shows an excellent agreement between tagged MRI and cine MRI with respect to left ventricular volumes and ejection fraction (LV-EF) (Table 1, Fig. 2). 
Fig. 1 a-c Tagged MRI images. c The endocardial contour was manually drawn in the endsystolic frame and a tracked backward to the end-diastolic frame. b Intermediary frame during systole. d, e The endocardial contours obtained by tagged MRI are superimposed over the corresponding standard cine MRI d end-diastolic and $\mathbf{e}$ end-systolic images
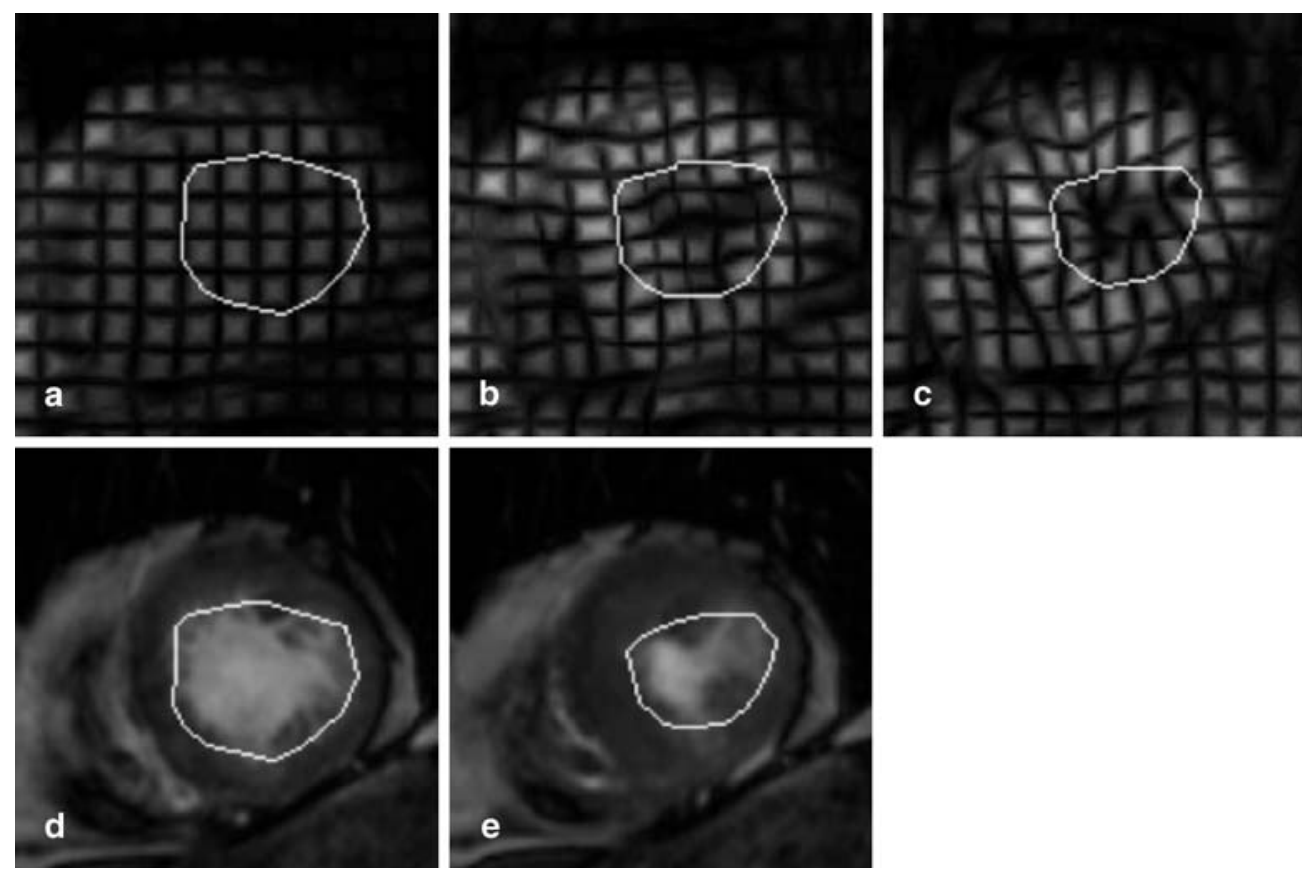

Table 1 Comparison between cine MRI and tagged MRI (in all observations $n=29$ including patients ( $n=9$ ) and volunteers ( $n=20$ ); $E S V$ end-systolic volume, $E D V$ end-diastolic volume, $E F$ ejection fraction, $C I$ confidence interval, $S D$ standard deviation, $N S$ not significant)

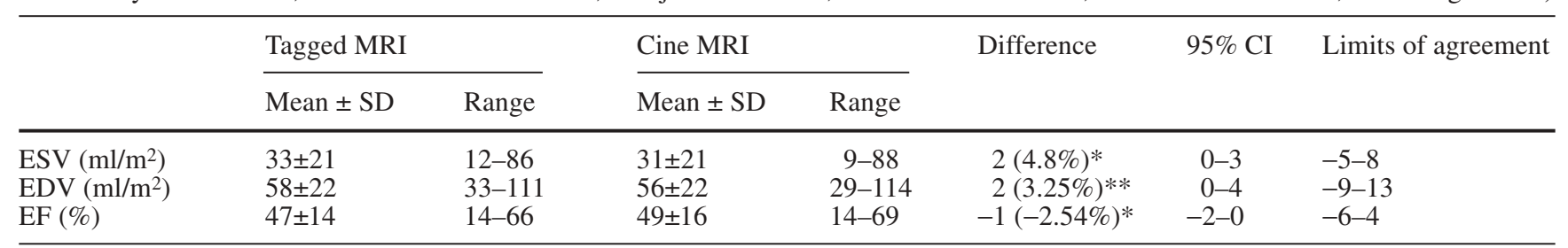

$* p<0.03 ; * * p=\mathrm{NS}$

The intra-observer variability for LV-EF assessment was low $\left(\mathrm{CV}_{\text {intra }}=0.4 \%\right)$. The first LV-EF measurement ranged from 14 to $53 \%$ with a mean of $30 \pm 13 \%$. The second LV-EF measurement ranged from 14 to $54 \%$ with a mean of $30 \pm 13 \%$. The high intra-observer agreement was reflected by a strong correlation $(y=1.0065 x-0.168, r>0.99, p=0.6)$ between the two measurements.

The inter-observer variability for LV-EF assessment was low $\left(\mathrm{CV}_{\text {inter }}=1.3 \%\right)$. LV-EF measured by the first observer ranged from 14 to $53 \%$ with a mean of $30 \pm 13 \%$. LV-EF measured by the second observer ranged from 14 to $53 \%$ with a mean of $29 \pm 13 \%$. A strong correlation $(y=1.0004 x-0.2861, r>0.99)$ was found between the measurements of the two observers and no statistical significant differences were observed.

\section{Discussion}

This is the first study to demonstrate that the assessment of global LV function by tagged MRI is accurate and reproducible and can be used to determine the prognosis and the effect of therapeutical intervention in heart failure patients. The use of a single imaging session to determine both global and regional LV function enables the optimal use of MRI scanning time and leads to improved patient comfort.

With tagged MRI, an overestimation of approximately $2 \mathrm{ml}$ was observed for both EDV and ESV. This can be explained by the fact that the endocardial contour may not exactly represent the true endocardial boundary. Indeed, tracking of the endocardial area with HARP was performed by copying the ES contour to the preceding frame, and then translating its vertices to equivalent positions relative to the MR tagging grid [13]. The same process was then repeated until the ED frame had been 

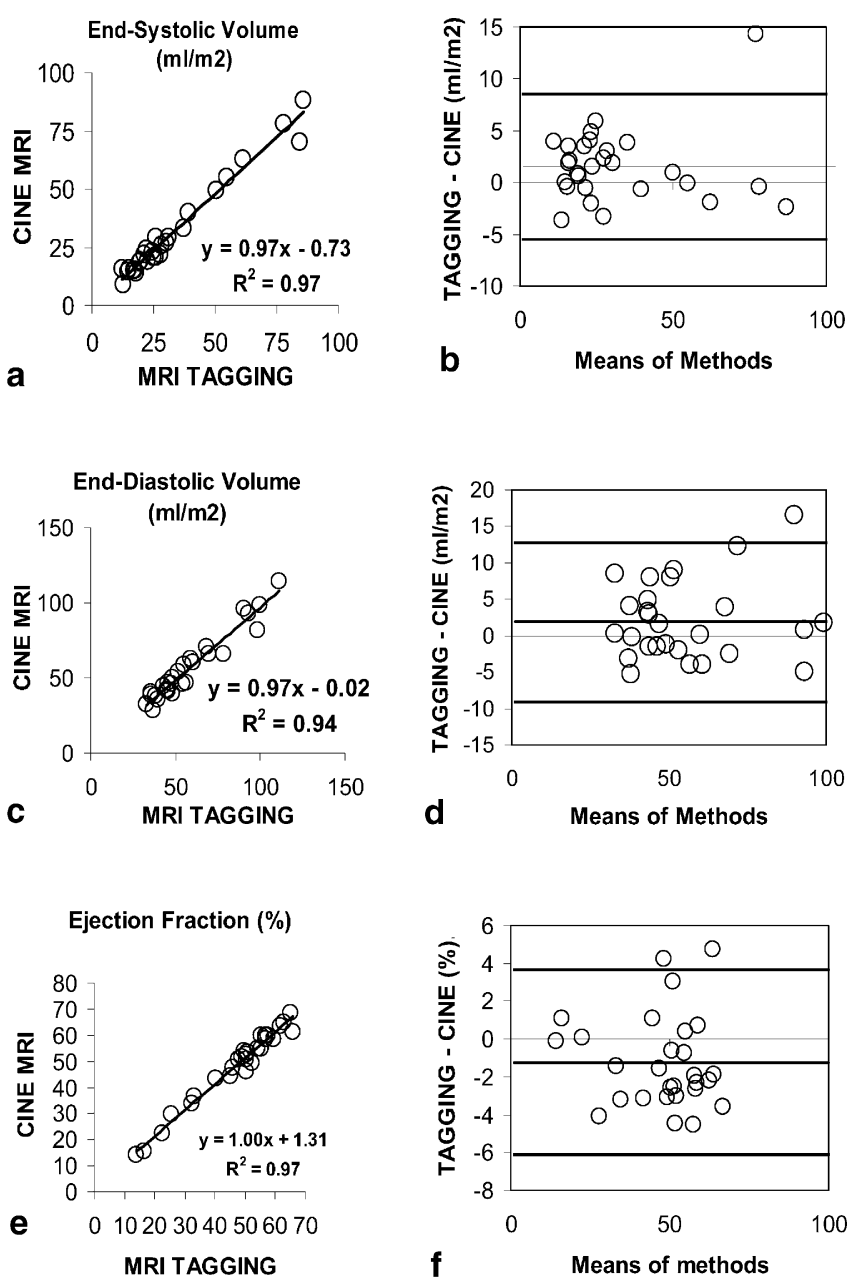

Fig. 2a-f Linear regression plots (a, c, e) and Bland-Altman analysis of agreement (plots) (b, d, f; solid line mean, dashed line mean $\pm 2 \mathrm{SD}$ ) between cine MRI and tagged MRI in the 28 subjects. a, b End-diastolic volume; c, d, end-systolic volume; $\mathbf{e}, \mathbf{f}$, ejection fraction

analysed. Tracking of the endocardial contour sometimes failed when the initial ES contour was drawn exactly at the endocardial cavity boundary. Subsequently, when the polygon vertices, which were copied to the preceding frame, are positioned within the ventricular cavity, rather than the myocardium, the program cannot properly detect the endocardial contour. To overcome this problem the initial contour must be drawn just inside the myocardium (1-2 mm) (Fig. 1e). In addition, since a relatively large delay between two consecutive frames in our study has contributed to this phenomenon, the use of MRI systems that can acquire more frames per cardiac cycle will further reduce the overestimation of myocardial volumes.

The difference between LV-EF obtained by tagged MRI and standard cine MRI was approximately $-1 \%$, which is explained by the overestimation of both EDV and ESV. However, a strong correlation and consistency between the two methods for the calculation of LV-EF indicates the existence of a systematic error. This small discrepancy between the LV-EF derived with cine and tagged MRI is of limited clinical importance, since changes in LV-EF within a patient over time are used for the evaluation of a therapeutical intervention. However, when LV-EF is used as a prognostic marker, correction of this systematic error may be indicated.

In this study, dark-blood tagged sequence, which can be used to enhance myocardium-cavity contrast, was not used for the following reasons. First, HARP analysis does not required cavity segmentation. Second, adding dark-blood preparation to the tagged cine sequence would increase scanning time and require longer breath-hold periods, which is a drawback in patients with dyspnea due to heart failure. Currently, the breathhold duration was about $15 \mathrm{~s}$, which was well supported by the patient, and enabled the acquisition of a complete slice.

The main study limitation is related to the small number of patients suffering from myocardial infarct, while other pathologies were not included. The study results therefore cannot be generalized to any clinical situation without caution.

\section{Conclusion}

This study demonstrates that tagged MRI is an accurate and highly reproducible method for assessment of LV$\mathrm{EF}$, which can be used as a prognostic tool in patients with LV dysfunction. In addition, high reproducibility allows for the detection of small changes in LV-EF over time to monitor the effect of therapeutical intervention in these patients. Since a single tagged MRI acquisition provides both regional and global LV function, available scanning time is optimally used. Future use of optimised tagged MRI sequences, such as segmented $k$-space acquisition, may reduce imaging time, which may further improve cost-effectiveness.

Acknowledgements We thank Rosalind Perrin for reviewing the paper and Dominique Joliat (Philips Medical Systems) for technical support. This study was partially supported by the Swiss National Science Foundation (grant SNF 31-57020.99) and the Geneva University Hospital (HUG). 


\section{References}

1. Haider AW, Larson MG, Benjamin EJ, Levy D (1998) Increased left ventricular mass and hypertrophy are associated with increased risk for sudden death. J Am Coll Cardiol 33:1454-1459

2. CIBIS-II Investigators and Committees (1999) The cardiac insufficiency bisoprolol study II (CIBIS-II): a randomized trial. Lancet 353:9-13

3. Bouton S, Yang A, McCrindle BW, Kidd L, McVeigh ER, Zerhouni EA (1991) Differentiation of tumor from viable myocardium using cardiac tagging with MR imaging. J Comput Assist Tomogr 15:676-678

4. Geskin G, Kramer CM, Rogers WJ, et al. (1998) Quantitative assessment of myocardial viability after infarction by dobutamine magnetic resonance tagging. Circulation 98:217-223

5. Vaduganathan P, He ZX, Vick GW III, Mahmarian JJ, Verani MS (1999) Evaluation of left ventricular wall motion, volumes, and ejection fraction by gated myocardial tomography with technetium $99 \mathrm{~m}$-labeled tetrofosmin: a comparison with cine magnetic resonance imaging. $\mathrm{J}$ Nucl Cardiol 6:3-10
6. Missouris CG, Forbat SM, Singer DRJ Markandu ND, Underwood R, MacGregor G (1996) Echocardiography overestimates left ventricular mass: a comparative study with magnetic resonance imaging in patients with hypertension. J Hypertens 14:1005-1010

7. Croisille P, Revel D (2000) MR imaging of the heart: functional imaging. Eur Radiol 10:7-11

8. Bogaert J, Maes A, Van de Werf F et al (1999) Functional recovery of subepicardial myocardial tissue in transmural myocardial infarction after successful reperfusion: an important contribution to the improvement of regional and global left ventricular function. Circulation 99:36-43

9. Zerhouni EA, Parish DM, Rogers WJ, Yang A, Shapiro EP (1988) Human heart: tagging with MR imaging. A method for noninvasive assessment of myocardial motion. Radiology 169:59-63

10. Axel L, Dougherty L (1989) Heart wall motion: improved method of spatial modulation of magnetization for MR imaging. Radiology 172:349-350

11. Gyngell ML (1988) The application of steady-state free precession in rapid 2DFT NMR imaging: FAST and CE-FAST sequences. Magn Reson Imaging 6:415-419
12. McVeigh ER (1996) MRI of myocardial function: motion tracking techniques. Magn Reson Imaging 14:137-150

13. Osman NF, Kerwin WS, McVeigh ER, Prince JL (1999) Cardiac motion tracking using CINE harmonic phase (HARP) magnetic resonance imaging. Magn Reson Med 42:1048-1060

14. Van der Geest RJ, Jansen E, Buller VGM, Reiber JHC (1997) Automated detection of left ventricular epi- and endocardial contours in short-axis MR images. Comput Cardiol 21:33-36

15. Pattynama PMT, Lamb HJ, Van der Velde EA, Van der Wall EE, De Roos A (1993) Left ventricular measurements with cine and spin-echo MR imaging; a study of reproducibility with variance component analysis. Radiology 187:261-268

16. Bland JM, Altman DG (1986) Statistical methods for assessing agreement between two methods of clinical measurement. Lancet 1:307-311 